\title{
Control of redundant joint structures using image information during the tracking of non-smooth trajectories
}

\author{
Gonzalo Lorenzo ${ }^{1}$, Jorge Pomares ${ }^{* 2}$, Asuncion Lledó ${ }^{3}$, Carlos A. Jara ${ }^{2}$ \\ 1. Department of General and Specific Didactics \\ 2. Department of Physics, System Engineering and Signal Theory \\ 3. Department of Developmental and Educational Psychology \\ *Corresponding author. Tel.: +34 965903400 E-mail address: jpomares@ua.es. \\ University of Alicante, Alicante, Spain
}

\begin{abstract}
Visual information is increasingly being used in a great number of applications in order to perform the guidance of joint structures. This paper proposes an image-based controller which allows the joint structure guidance when its number of degrees of freedom is greater than the required for the developed task. In this case, the controller solves the redundancy combining two different tasks: the primary task allows the correct guidance using image information, and the secondary task determines the most adequate joint structure posture solving the possible joint redundancy regarding the performed task in the image space. The method proposed to guide the joint structure also employs a smoothing Kalman filter not only to determine the moment when abrupt changes occur in the tracked trajectory, but also to estimate and compensate these changes using the proposed filter. Furthermore, a direct visual control approach is proposed which integrates the visual information provided by this smoothing Kalman filter. This last aspect permits the correct tracking when noisy measurements are obtained. All the contributions are integrated in an application which requires the tracking of the faces of Asperger children.
\end{abstract}

Keywords: service robots, robot vision, image-based control, control of joint structures

\section{Introduction}

Robots are becoming increasingly integrated into the lives of ordinary people. In the last years, different research works are being developed in order to apply robots, not only in industrial factories, but also in human workspaces. This requires providing the robots with the capability to carry out fluent mobility in unstructured and changing environments. This fact can be solved adding adequate 
sensor technology like visual information to adapt the robot motion into a dynamic workspace [1]. Currently, there are a great number of previous works which propose the use of computer vision to perform the guidance of vehicles, robots, etc. [2]. As shown in this last paper, the information provided by a camera is adequate not only to supervise a task but also to perform the guidance of robots or redundant joint structures. Classical visual control systems generate velocity references to perform the guidance of joint structures. Thus, internal joint controllers are required in order to guarantee the correct joint behavior and to achieve the velocity reference. Therefore, a hierarchical architecture with two nested control loops is employed: the external loop implements the visual feedback and the internal loop the joint control. In previous works (see e.g. [3]), direct controllers are proposed which performs the joint control directly using visual information. In this case, the visual feedback generates the torques to be applied to the joints. By using this kind of controllers, the system reacts more quickly to abrupt changes in the trajectory and the visual information is employed directly to stabilize the joint structure. In this paper, a modification of these direct controllers is proposed not only to perform the joint structure guidance, but also to simultaneously perform the postural control determining the appropriate joint configuration.

Although visual information is extensively employed to perform the guidance of joint structures and serial robots, this kind of information presents several disadvantages. On one hand, it requires the previous use of computer vision algorithms to extract the visual features employed by the visual controller. On the other hand, the precision of these extracted features depends on several factors such as illumination, intrinsic parameters estimation, image resolution, etc. Therefore, a noisy signal can be obtained from the previous mentioned computer vision algorithms which will be employed by the visual controllers to perform the required tasks. In some workspaces, several authors propose combining the visual information with other sensors in order to improve the system guidance. As example, the hybrid visual/force control systems propose the simultaneous control of separate directions. A position control is carried out using the visual information and a force control is developed employing each controller in different directions [4] [5]. In this paper, a smoothing filter is integrated in the proposed direct controller in order to reduce the effect of the mentioned noise.

Kalman filters [6] are the most used technique to implement Bayes filters [7]. The standard Kalman filter approximates the beliefs of the Bayes filter by Gaussian distributions and represents the state transition model and the observation model as linear stochastic relations of the state. Previous approaches have applied Kalman filters for human tracking. In [8] a complementary Kalman filter is proposed in a navigation system for tracking a pedestrian using inertial sensors and a GPS receiver. This complementary Kalman filter estimates sensor errors instead of direct measurements. This kind of Kalman filter is also proposed in [9] that employs the differences between an inertial tracking system and a magnetic tracker. Caron et al. [10] extend the definition of the Kalman filter to include multiple sensors, where each sensor is weighted according to fuzzy context variables. The work described in [11] employs the extended Kalman filter in a tracking system composed by a camera and 
an inertial system. The main contribution of the presented tracker is that it generates a smooth estimation of the obtained image measurements in order to be integrated in the proposed direct visual controller.

The objective of most image-based visual servoing systems is to achieve a desired image configuration from an initial one [2][12]; however, using the approach described in this paper, the robot must track a given image trajectory. Many articles have focused on the design of visual servoing systems to track image trajectories, especially in the field of autonomous vehicle guidance [13][14]. Furthermore, there are visual servoing systems to track time-independent image trajectories and to track time-dependent image trajectories. In the first case, the image trajectory is defined as a set of image configurations that the robot must track and no time constraints are considered [15]. In the second case, the desired image trajectory to be tracked is indicated as a time-dependent function [14][16]. In our case, the time constraints must be taken into account; therefore, a time-dependent approach will be defined. Only few works address the problem of controlling a robot manipulator to track desired image trajectories taking explicitly into account the robot dynamics (see e.g. [3][17][18]). Within these last works, only few approaches such as [3][19] carry out the tracking of time-dependent trajectories and none of these previous works exploit the robot redundancy to simultaneously perform different tasks such as the tracking and the robot postural control.

In this paper, the employed joint structure is a serial robot with 7 degrees of freedom (dof) which is redundant regarding the task performed in the image space. There are previous controllers which employ visual information to simultaneously guide a robot and solve its redundancy (see e.g. [20][21][22]). However, these previous works do not consider the robot dynamics. Therefore, a direct visual servoing approach is not employed. Only few works consider dynamic properties during the redundancy resolution (see e.g. [23][24][25]) and none of these previous works solves the redundancy to simultaneously carry out different tasks during the tracking of time-dependent trajectories. In this paper, a direct controller is proposed to track time dependent trajectories and the joint redundancy will be employed to accomplish prioritized tasks, where the higher priority task is guaranteed and is called the primary task. The primary task will be used to perform the joint structure guidance employing the proposed direct visual controller. The secondary task is accomplished taken advantage of the redundancy and is only achieved when the primary task is guaranteed. This secondary task will be employed to perform the postural control of the joint structure. Therefore, the proposed direct controller is able to simultaneously guide the robot, tracking the desired trajectory, and control the most appropriate joint configuration.

As is previously indicated, a redundant joint structure with and eye-in-hand camera system will be applied to track noisy image trajectories. When a noisy image trajectory is considered, the detection of abrupt changes can be useful. For example, in the task considered in this paper, the eyein-hand camera system is employed to detect [26] and to perform the tracking of a child's face. The detection of an abrupt change is required to determine when the children perform anomalous motions. 
Additionally, the proposed method is not only able to detect abrupt changes but also to estimate and compensate these changes using the proposed filter.

This paper is organized as follows: Section 2 describes the system architecture. In Section 3, the visual servo control of the robot system is described. Section 4 shows the dynamic aspects of the proposed controller. Section 5 presents the simulation and experimental results obtained. Finally, some conclusions are discussed in Section 6.

\section{System architecture and application}

In this paper, the proposed controllers will be employed in order to guide a 7 dof serial robot and simultaneously solve its redundancy during the tracking of noisy image trajectories. In terms of practical use, these controllers can be employed in a wide range of industrial applications such as polishing, milling or cutting where the trajectory tracked by the robot is specified in the image space. However, in this paper the workspace where the presented controllers will be applied consists of the tracking of the face of Asperger children. These children are characterized by problems with the use of language with a communicative purpose, nonverbal communication difficulties, specific interests and visual thinking. They have problems in social interaction and difficulties to express emotions. However, they generally present a good level of intelligence with excellent memory in terms of data collection. In order to improve the social skills of these students, an immersive virtual reality system will be employed. This system will be used to simulate real environments where the Asperger children have a chance to practice several social skills in a safe and controlled environment. In this workspace, the face tracking and recognition is required in order to detect the student's mood (and possible abrupt changes in the face trajectory) and accordingly update the virtual environment (the pedagogical aspects of this study have been previously published in [27]).

To do this, a robot with an eye-in-hand camera system is employed as is shown in Figure 1. The employed robot is a 7 dof Mitsubishi PA-10 robotic manipulator. The Mitsubishi PA-10 robotic manipulator has an eye-in-hand PHOTONFOCUS MV-D752-160-CL-8 camera installed at its endeffector. The camera is able to acquire and to process up to 100 frames/second using an image resolution of $320 \times 240$. 


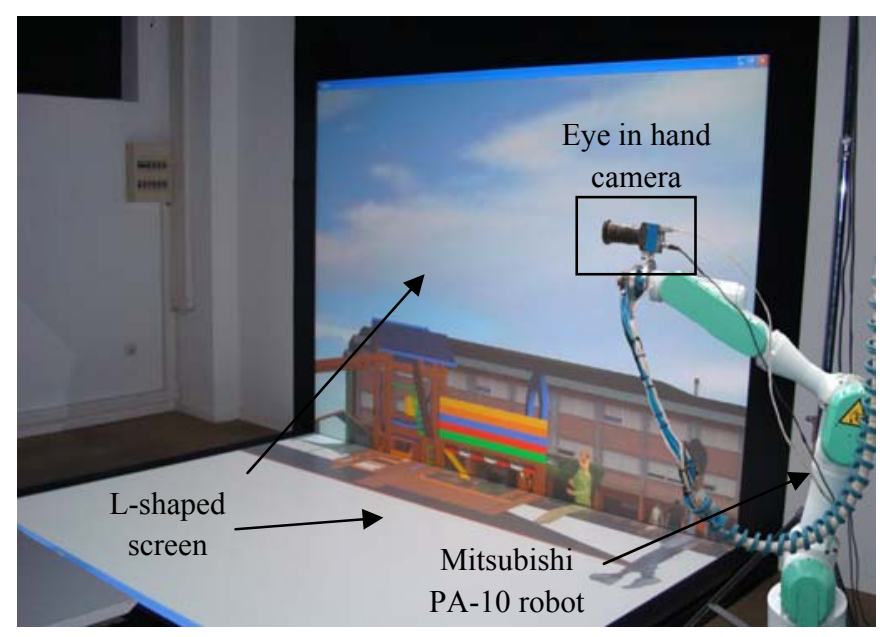

Figure 1. System architecture

\section{Robotic platform for tracking faces}

\subsection{Visual servoing}

In order to perform the tracking of the child's face, the previously indicated $n=7$ dof robot is employed with a camera located at its end-effector. The robot carries out a continuous tracking in order to guarantee that the face is always in the camera field. This section describes the visual servoing strategy employed to perform the tracking.

First, we consider $\mathbf{q} \in \mathfrak{R}^{\mathrm{nx} 1}$ as the set of joint positions, $\dot{\mathbf{q}} \in \mathfrak{R}^{\mathrm{nx} 1}$ represents the joint velocities vector and $\ddot{\mathbf{q}} \in \mathfrak{R}^{\mathrm{nx} 1}$ are the joint accelerations. As previously indicated, the robot is guided by visual information. In a general case, this visual information is a vector of $\mathrm{k}$ image point features $\mathbf{s}=\left[f_{1 \mathrm{x}}, f_{1 \mathrm{y}}, f_{2 \mathrm{x}}, f_{2 \mathrm{y}}, \ldots, f_{\mathrm{kx}}, f_{\mathrm{ky}}\right]^{\mathrm{T}} \in \mathfrak{R}^{2 \mathrm{k}}$, which are extracted from the child's face. The following relation is established by the interaction matrix $\mathbf{L}_{\mathrm{s}}[1]$ :

$$
\dot{\mathbf{s}}=\mathbf{L}_{\mathrm{s}}(\mathbf{r}) \dot{\mathbf{r}}_{\mathrm{c}}
$$

where $\dot{\mathbf{s}}$ is the time derivative of the image features, $\mathbf{r}_{\mathrm{c}}$ represents the pose of the camera located at the robot end-effector and $\dot{\mathbf{r}}_{\mathrm{c}}$ is the corresponding camera velocity. The robot-end is performing a tracking in an m-dimensional space, therefore, the end-effector pose $\mathbf{r}_{\mathrm{c}} \in \mathfrak{R}^{\mathrm{m}}$. The robot Jacobian relates the end-effector robot velocity and the joint-space velocity $\dot{\mathbf{q}}$ :

$$
\dot{\mathbf{r}}_{\mathrm{c}}=\mathbf{J}_{\mathrm{r}}(\mathbf{q}) \dot{\mathbf{q}}
$$

From (1) and (2) the following relation can be established that relates the joint and the image space:

$$
\dot{\mathbf{s}}=\mathbf{L}_{\mathrm{s}}(\mathbf{r}) \mathbf{J}_{\mathrm{r}}(\mathbf{q}) \dot{\mathbf{q}}=\mathbf{J}(\mathbf{q}) \dot{\mathbf{q}}
$$


where $\mathbf{J}=\mathbf{L}_{\mathrm{s}}(\mathbf{r}) \mathbf{J}_{\mathrm{r}}(\mathbf{q})=\mathbf{J}(\mathbf{q}) \in \mathfrak{R}^{2 \mathrm{k} \times \mathrm{n}}$. Differentiating (3) with respect to the time, the following expression can be obtained:

$$
\ddot{\mathbf{s}}=\mathbf{J} \ddot{\mathbf{q}}_{\mathrm{r}}+\dot{\mathbf{J}} \dot{\mathbf{q}}
$$

where $\ddot{\mathbf{s}}$ denotes the image acceleration. An acceleration based controller will be proposed in the joint space, therefore, the joint accelerations are denoted as $\ddot{\mathbf{q}}_{\mathrm{r}}$ in (4) to represent the resolved acceleration which will be applied by the controller.

In order to perform the visual control task taking into account the robot redundancy, two different tasks are defined:

- Primary task. This task consists of the joint structure guidance (in this case the tracking of the child's face). This task will be the higher priority task.

- Secondary task. Taking advantage of the robot redundancy, a secondary task can be established so that this task will be only satisfied when the correct tracking (primary task) is achieved. The joint structure is redundant regarding the required task in the image space. Therefore, a desired joint configuration is indicated by the user, $\mathbf{q}_{\mathrm{u}}$. This secondary task is used in order to maintain the joint configuration closest to the desired one with the prerequisite that the tracking is satisfied.

In order to accomplish the two tasks, the required joint accelerations, $\ddot{\mathbf{q}}_{\mathrm{r}}$, can be obtained from (4), thereby ensuring correct tracking of the faces:

$$
\ddot{\mathbf{q}}_{\mathrm{r}}=\mathbf{J}^{+}\left(\ddot{\mathbf{s}}_{\mathrm{r}}-\dot{\mathbf{J}} \dot{\mathbf{q}}\right)
$$

This last equation does not consider redundancy resolution. The basic idea for redundancy resolution at the acceleration level is to compute the desired joint accelerations integrating the secondary task in the nullspace of $\mathbf{J}$. This last approach can be achieved by employing this expression:

$$
\ddot{\mathbf{q}}_{\mathrm{r}}=\mathbf{J}^{+}\left(\ddot{\mathbf{s}}_{\mathrm{r}}-\dot{\mathbf{J}} \dot{\mathbf{q}}\right)+\left(\mathbf{I}-\mathbf{J}^{+} \mathbf{J}\right) \cdot\left(-\mathbf{K}_{\mathrm{Dp}} \dot{\mathbf{q}}-\alpha \nabla \mathbf{u}\right)
$$

where the parameter $\alpha$ is a positive gain and $\mathbf{I}-\mathbf{J}^{+} \mathbf{J}$ projects the gradient of $\mathbf{u}$ with a damping term in the joint space $\left(-\mathbf{K}_{\mathrm{Dp}} \dot{\mathbf{q}}\right)$ onto the null space of the Jacobian $\mathbf{J}\left(\mathbf{K}_{\mathrm{Dp}}>0\right)$. Finally, $\mathbf{u}$ is a cost function to be minimized under the constraint that the tracking defined in the primary task is satisfied:

$$
\mathbf{u}=\frac{1}{2}\left(\mathbf{q}-\mathbf{q}_{\mathrm{u}}\right)^{\mathrm{T}} \mathbf{K}_{\mathrm{u}}\left(\mathbf{q}-\mathbf{q}_{\mathrm{u}}\right)
$$

where $\mathbf{K}_{\mathrm{u}}$ is a diagonal matrix with positive weights. The acceleration in the image space, $\ddot{\mathbf{s}}_{\mathrm{r}}$, to perform the tracking can be obtained as:

$$
\ddot{\mathbf{s}}_{\mathrm{r}}=\ddot{\mathbf{s}}_{\mathrm{d}}+\mathbf{K}_{\mathrm{D}}\left(\dot{\mathbf{s}}_{\mathrm{d}}-\dot{\mathbf{s}}\right)+\mathbf{K}_{\mathrm{P}}\left(\mathbf{s}_{\mathrm{d}}-\mathbf{s}\right)=\ddot{\mathbf{s}}_{\mathrm{d}}+\mathbf{K}_{\mathrm{D}} \dot{\mathbf{e}}_{\mathrm{s}}+\mathbf{K}_{\mathrm{P}} \mathbf{e}_{\mathrm{s}}
$$


where $\ddot{\mathbf{s}}_{\mathrm{d}}, \dot{\mathbf{s}}_{\mathrm{d}}$ and $\mathbf{s}_{\mathrm{d}}$ are the desired image space accelerations, velocities and positions, respectively. $\mathbf{K}_{\mathrm{P}}$ and $\mathbf{K}_{\mathrm{D}}$ are the PD gain matrices. Furthermore, $\mathbf{e}_{\mathrm{s}}=\mathbf{s}_{\mathrm{d}}-\mathbf{s}$, is the image error, $\dot{\mathbf{e}}_{\mathrm{s}}=\dot{\mathbf{s}}_{\mathrm{d}}-\dot{\mathbf{s}}$ is the time derivative of the image error, and $\ddot{\mathbf{e}}_{\mathrm{s}}=\ddot{\mathbf{s}}_{\mathrm{d}}-\ddot{\mathbf{s}}$ will be denoted as the acceleration error or second derivative of the image error.

\subsection{Smoothing filter with abrupt changes estimation}

Considering the previous described visual servoing system, it is possible to affirm that the image position, velocity and acceleration are necessary in order to perform the tracking. In this section, the main properties of the proposed smoothing filter are described. In our case, the software described in [28] is employed to determine the image position of the face at each iteration $\mathrm{i}, z_{(\mathrm{i})}=\left({ }^{\mathrm{i}} \mathrm{f}_{\mathrm{x}}\right.$, $\left.{ }^{\mathrm{i}} f_{\mathrm{y}}\right)^{\mathrm{T}}$. This last term is the measurement that it will be employed to update the Kalman filter which will be described in the next paragraphs (the filter is used to estimate the current state, iteration $i$, from the previous measurements). To implement the Kalman filter, authors consider $x_{(\mathrm{i})}$ as the state vector which includes the position, velocity and acceleration in the image space of the child at iteration $i$. The evolution of the sequence of states $x_{(\mathrm{i})}$ is given by:

$$
x_{(\mathrm{i})}=g_{\mathrm{i}}\left(x_{(\mathrm{i}-1)}, v_{(\mathrm{i}-1)}\right)
$$

where $x_{(\mathrm{i}-1)}$ is the state vector in the iteration $\mathrm{i}-1, g_{\mathrm{i}}: \mathfrak{R}^{\mathrm{nx}} \times \mathfrak{R}^{\mathrm{nv}} \rightarrow \mathfrak{R}^{\mathrm{nx}}$ is a function of the state $x_{(\mathrm{i}-1)}$ and $v_{(\mathrm{i}-1)}$ is the state noise. The terms $\mathrm{nx}$ and $\mathrm{nv}$ are the dimensions of the state vectors and process noise, respectively. We suppose that $v$ is a random variable with normal distribution and covariance matrix $\mathbf{Q}$. The covariance matrix $\mathbf{Q}$ is commonly referred to as the process noise covariance matrix. It accounts for possible changes in the process between two consecutive iterations that are not already accounted for in the state transition matrix.

The tracking objective is recursively estimate $x_{(\mathrm{i})}$ using the measurements:

$$
\mathrm{z}_{(\mathrm{i})}=h_{\mathrm{i}}\left(x_{(\mathrm{i})}, w_{(\mathrm{i})}\right)
$$

where $h_{\mathrm{i}}: \mathfrak{R}^{\mathrm{nx}} \times \mathfrak{R}^{\mathrm{nn}} \rightarrow \mathfrak{R}^{\mathrm{nz}}$ is a function of the state $x_{(\mathrm{i})}$ and $w_{(\mathrm{i})}$ is the measurement noise (nz and nn are the dimensions of the measurement and the measurement noise, respectively). The measurement noise is a random variable with normal distribution and covariance matrix $\mathbf{R}$. This matrix quantifies the precision of the measurements obtained by using the computer vision system. The relation between $\mathbf{Q}$ and $\mathbf{R}$ determines the results obtained by using the Kalman filter. The value of $\mathbf{R}$ must be high (compared to $\mathbf{Q}$ ) when a noisy measurement is obtained. Figure 2 shows the effect obtained when the relation between $\mathbf{Q}$ and $\mathbf{R}$ varies. This figure represents by means of squares the real motion of a point in a plane and by means of circles the measurements of this motion (a noisy measurement is considered). A Kalman filter is applied to estimate the point motion. The estimations obtained when 
$\mathbf{Q}=10^{-2} \mathbf{I}, \mathbf{R}=3 \cdot 10^{-1} \cdot \mathbf{I}$ are represented in green, when $\mathbf{Q}=10^{-2} \cdot \mathbf{I}, \mathbf{R}=3 \cdot 10^{-2} \cdot \mathbf{I}$ are indicated in red and when $\mathbf{Q}=10^{-2} \cdot \mathbf{I}, \mathbf{R}=3 \cdot 10^{-3} \cdot \mathbf{I}$ are indicated in blue. As this figure shows, when $\mathbf{R}$ is high compared to $\mathbf{Q}$, the estimations are less affected by the noise. However, the estimations are not close to the measurements (more iterations will be required to track abrupt motions). In the cases when the measurements are not noisy, the values of $\mathbf{Q}$ and $\mathbf{R}$ indicated in Figure 2.c will be more adequate in order to react more adequately depending on the object measurements. A study about the effect of varying these matrices in image tracking applications can be seen in [29]. The choice of the filter $\mathbf{Q}$ and $\mathbf{R}$ matrices still poses a major challenge and adaptive approaches are under research [30]. In the result section these matrices are experimentally determined.

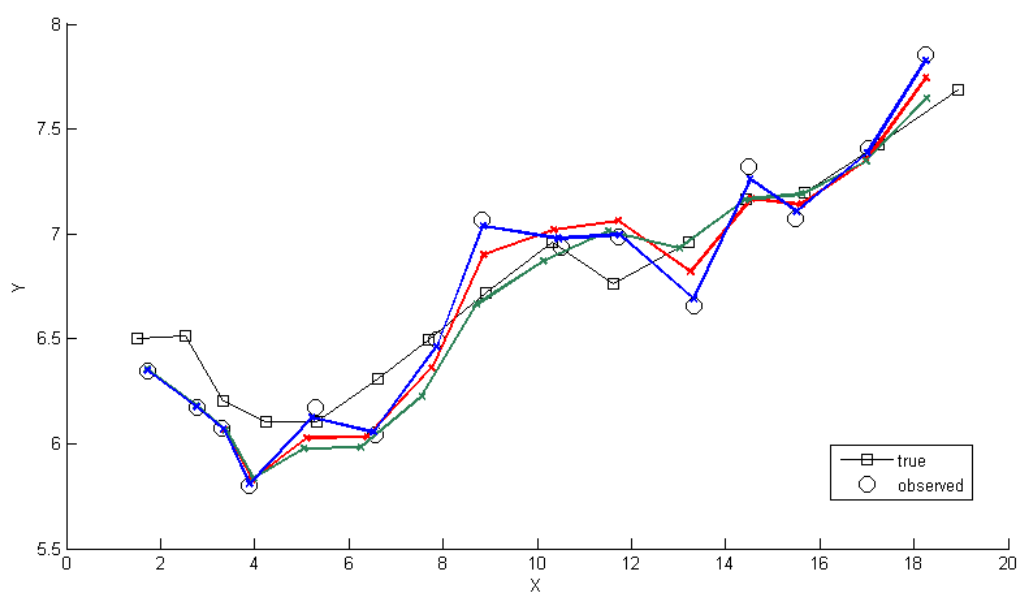

Figure 2. Kalman filter when the relation between $\mathbf{Q}$ and $\mathbf{R}$ varies. a) $\mathbf{Q}=10^{-2} \cdot \mathbf{I}, \mathbf{R}=3 \cdot 10^{-1} \cdot \mathbf{I}$ (green), b) $\left.\mathbf{Q}=10^{-2} \cdot \mathbf{I}, \mathbf{R}=3 \cdot 10^{-2} \cdot \mathbf{I}(\mathrm{red}), \mathrm{c}\right) \mathbf{Q}=10^{-2} \cdot \mathbf{I}, \mathbf{R}=3 \cdot 10^{-3} \cdot \mathbf{I}$ (blue).

Considering the previous assumptions, the equations of the state and measurement models of the Kalman filter that allows to estimate $x$ using the set of measurements $z_{(1 \mid \mathrm{i})}=\left\{z_{(\mathrm{j})}, j=1, \ldots, \mathrm{i}\right\}$, are:

$$
\begin{aligned}
& x_{(\mathrm{i}+1)}=\mathbf{F} \cdot x_{(\mathrm{i})}+v_{(\mathrm{i})} \\
& z_{(\mathrm{i})}=\mathbf{H} \cdot x_{(\mathrm{i})}+w_{(\mathrm{i})}
\end{aligned}
$$

where:

- $\mathbf{F}$ is the state transition matrix which relates the state in the previous step with the current state (in absence of noise).

- $\quad \mathbf{H}$ is the measurement matrix which relates the state with the measurement $z_{(\mathrm{i})}$.

The state transition and measurement matrices $(\mathbf{F}$ and $\mathbf{H})$ are: 


$$
\mathbf{F}=\left[\begin{array}{cc}
\mathbf{A} & 0 \\
0 & \mathbf{A}
\end{array}\right], \mathbf{A}=\left[\begin{array}{ccc}
1 & \Delta T & 1 / 2 \Delta T^{2} \\
0 & 1 & \Delta T \\
0 & 0 & 1
\end{array}\right], \mathbf{H}=\left[\begin{array}{cccccc}
1 & 0 & 0 & 0 & 0 & 0 \\
0 & 0 & 0 & 1 & 0 & 0
\end{array}\right]
$$

where $\Delta T$ represents the time between successive video frames.

Using the Kalman filter, the following variables can be obtained: the prediction of the vector state $x_{(i+1 \mid i)}$, the covariance of the prediction error $\mathbf{P}_{(i+1 \mid i)}$, the estimation of the vector state $x_{(i+1 \mid i+1)}$ and the covariance of the estimation error $\mathbf{P}_{(\mathrm{i}+1 \mid \mathrm{i}+1)}$. This information is obtained from the estimation of the vector state $x_{(\mathrm{i} i \mathrm{i})}$ and the covariance of the estimation error $\mathbf{P}_{(\mathrm{i} i \mathrm{i})}$ in the previous estimation. More details of the Kalman filter can be seen in [31].

In order to compute the smoothing solution, $x_{(i+1 \mid i+1)}^{\mathrm{S}}$, for the previous described model, the following equations are defined:

$$
\begin{gathered}
\mathbf{L}_{(\mathrm{i}+1)}=\mathbf{P}_{(\mathrm{i} \mid \mathrm{i})} \cdot \mathbf{F}^{\mathrm{T}} \cdot \mathbf{P}_{(\mathrm{i}+1 \mid \mathrm{i})}^{-1} \\
x_{(\mathrm{i}+1 \mathrm{i}+1)}^{\mathrm{S}}=x_{(\mathrm{ii})}+\mathbf{L}_{(\mathrm{i}+1)} \cdot\left(x_{(\mathrm{i}+1 \mathrm{i}+1)}-x_{(\mathrm{i}+1 \mathrm{i})}\right) \\
\mathbf{P}_{(\mathrm{i}+1 \mid \mathrm{i}+1)}^{\mathrm{S}}=\mathbf{P}_{(\mathrm{i}+1 \mid \mathrm{i})}+\mathbf{L}_{(\mathrm{i}+1)} \cdot\left(\mathbf{P}_{(\mathrm{i}+1 \mid \mathrm{i}+1)}-\mathbf{P}_{(\mathrm{i}+1 \mid \mathrm{i})}\right) \cdot \mathbf{L}_{(\mathrm{i}+1)}^{\mathrm{T}}
\end{gathered}
$$

As is previously indicated, the state vector $x$ has 6 components (position, velocity and acceleration in the image space).

In order to detect an abrupt change in a parameter represented in the state vector, a vector, $a$, must be defined by the user. This vector, which is defined previously to begin the tracking, has 6 components (one for each component of the state vector). A given component has the value of 1 if the system tries to detect abrupt changes in the corresponding magnitude, i.e., image position, velocity or acceleration in $\mathrm{x}$ and $\mathrm{y}$ directions ( 0 if the system does not detect changes in this magnitude). In this filter, the innovation reflects the discrepancy between the predicted value and the actual measurement. If there is not an abrupt change during the tracking, the innovation can be represented as:

$$
\gamma_{\text {no(i) }}=z_{(\mathrm{i})}-\mathbf{H} \cdot x_{(\mathrm{ili}-1)}^{\mathrm{S}}
$$

where $x_{(\mathrm{i} \mid \mathrm{i}-1)}^{\mathrm{S}}$ is the a priori estimate (prediction) at step i from the previous value of $x$.

Now, we consider that there is a change in the magnitude and direction indicated by the vector $a$. The size of the change is denoted by $\phi$. Considering this abrupt change, the new innovation can be expressed as: 


$$
\gamma_{(\mathrm{i})}=\gamma_{\mathrm{no}(\mathrm{i})}+b(\mathrm{i} ; \theta) \cdot \phi
$$

where $b(\mathrm{i} ; \theta)$ is the effect (on the innovation measured at the iteration i) of the previous mentioned change in the face trajectory that is produced at the iteration $\theta(\theta<\mathrm{i})$ :

$$
b(\mathrm{i} ; \theta)=a-j(\mathrm{i}-1 ; \theta)
$$

$j(\mathrm{i} ; \theta)$ is the effect of the change in the measured face state over the innovation value:

$$
j(\mathrm{i} ; \theta)=\mathbf{F} \cdot j(\mathrm{i}-1 ; \theta)+{ }^{\mathrm{i}} \mathbf{K} \cdot b(\mathrm{i} ; \theta)
$$

where ${ }^{\mathrm{i}} \mathbf{K}$ is the gain of the Kalman filter. The objective of obtaining the previous value is to determine when an abrupt change is observed in the face. To obtain this information, the generalized likelihood ratio (GLR) is obtained from the previous variables. To obtain this parameter the following equations are applied:

$$
\operatorname{GLR}(\mathrm{i} ; \theta)=\frac{d^{2}(\mathrm{i} ; \theta)}{c(\mathrm{i} ; \theta)}
$$

where:

$$
c(\mathrm{i} ; \theta)=\sum_{\mathrm{m}=\theta}^{\mathrm{i}} \frac{b^{2}(\mathrm{~m} ; \theta)}{v_{(\mathrm{m})}}
$$

and:

$$
d(\mathrm{i} ; \theta)=\sum_{\mathrm{m}=\theta}^{\mathrm{i}} \frac{b(\mathrm{~m} ; \theta) \cdot \gamma_{(\mathrm{m})}}{v_{(\mathrm{m})}}
$$

In the result section, we have described the use of this ratio in order to detect situations where the child's face describes abrupt changes. As it will be shown, the value of the obtained ratio increases when abrupt changes are measured in the state of the child's face.

\section{Dynamic visual control with abrupt changes detection}

In classical visual servoing systems, the robot end-effector or joint velocities are obtained in order to position the camera with respect to a reference object. Once these velocities are obtained, the robot's internal controller generates the adequate joint torques to achieve the generated velocities. In this paper, the use of a direct visual servoing approach allows the system to directly generate the forces and torques to be applied in the robot joints in order to carry out the prioritized tasks as it was described in Section 3.1. Using this kind of controller, the tracking can be performed more accurately and the system reacts more quickly to abrupt changes in the trajectory. 
Using Equation (6), the required joint accelerations to track the desired image trajectory can be obtained. The control action for acceleration-based operational space controllers can be written as:

$$
\boldsymbol{\tau}=\mathbf{M}(\mathbf{q}) \ddot{\mathbf{q}}_{\mathrm{r}}+\mathbf{C}(\mathbf{q}, \dot{\mathbf{q}})+\mathbf{g}(\mathbf{q})
$$

where $\mathbf{M}(\mathbf{q}) \in \mathfrak{R}^{\mathrm{nxn}}$ is the symmetric positive definite manipulator inertia matrix, $\mathbf{C}(\mathbf{q}, \dot{\mathbf{q}}) \in \mathfrak{R}^{\mathrm{nx} 1}$ is the vector of centripetal and Coriolis torques and $\mathbf{g}(\mathbf{q})$ is the gravitational force. Finally, $\boldsymbol{\tau} \in \mathfrak{R}^{\mathrm{nx}}$ is a vector of applied joint torques. From (24) and (6), the direct visual controller is:

$$
\boldsymbol{\tau}=\mathbf{M}(\mathbf{q}) \mathbf{J}^{+}\left(\ddot{\mathbf{s}}_{\mathrm{r}}-\dot{\mathbf{J}} \dot{\mathbf{q}}\right)+\mathbf{M}(\mathbf{q})\left(\mathbf{I}-\mathbf{J}^{+} \mathbf{J}\right) \cdot\left(-\mathbf{K}_{\mathrm{Dp}} \dot{\mathbf{q}}-\alpha \nabla \mathbf{u}\right)+\mathbf{C}(\mathbf{q}, \dot{\mathbf{q}})+\mathbf{g}(\mathbf{q})
$$

Considering the previous assumptions, the closed loop behavior can be easily obtained as:

$$
\mathbf{M}(\mathbf{q}) \ddot{\mathbf{q}}_{\mathrm{r}}=\mathbf{M}(\mathbf{q}) \mathbf{J}^{+}\left(\ddot{\mathbf{s}}_{\mathrm{r}}-\mathbf{J} \dot{\mathbf{q}}\right)+\mathbf{M}(\mathbf{q})\left(\mathbf{I}-\mathbf{J}^{+} \mathbf{J}\right) \cdot\left(-\mathbf{K}_{\mathrm{Dp}} \dot{\mathbf{q}}-\alpha \nabla \mathbf{u}\right)
$$

By pre-multiplying $\mathbf{J}(\mathbf{M}(\mathbf{q}))^{-1}$ to both sides of (26):

$$
\begin{aligned}
& \mathbf{J} \ddot{\mathbf{q}}_{\mathrm{r}}=\ddot{\mathbf{s}}_{\mathrm{r}}-\dot{\mathbf{J}} \dot{\mathbf{q}} \\
& \mathbf{J} \ddot{\mathbf{q}}_{\mathrm{r}}+\dot{\mathbf{J}} \dot{\mathbf{q}}=\ddot{\mathbf{s}}_{\mathrm{r}}
\end{aligned}
$$

Considering (4), the left part of Equation (28), $\mathbf{J} \ddot{\mathbf{q}}_{\mathrm{r}}+\dot{\mathbf{J}} \dot{\mathbf{q}}$, is equal to $\ddot{\mathbf{s}}$, and using (8) the right part, $\ddot{\mathbf{s}}_{\mathrm{r}}$, is equal to $\ddot{\mathbf{s}}_{\mathrm{d}}+\mathbf{K}_{\mathrm{D}} \dot{\mathbf{e}}_{\mathrm{s}}+\mathbf{K}_{\mathrm{P}} \mathbf{e}_{\mathrm{s}}$. Therefore:

$$
\ddot{\mathbf{s}}=\ddot{\mathbf{s}}_{\mathrm{d}}+\mathbf{K}_{\mathrm{D}} \dot{\mathbf{e}}_{\mathrm{s}}+\mathbf{K}_{\mathrm{P}} \mathbf{e}_{\mathrm{s}} \rightarrow \ddot{\mathbf{e}}_{\mathrm{s}}+\mathbf{K}_{\mathrm{D}} \dot{\mathbf{e}}_{\mathrm{s}}+\mathbf{K}_{\mathrm{P}} \mathbf{e}_{\mathrm{s}}=0
$$

which achieves asymptotic tracking in the image space.

\section{Results}

A series of experiments were conducted to illustrate the performance of the different contributions proposed in the paper. The robotic architecture and the eye-in-hand camera system presented in Section 2 were employed. The camera is supposed to be previously calibrated and the camera intrinsic parameters are $\left(\mathrm{u}_{0}, \mathrm{v}_{0}\right)=(298,225) \mathrm{px}$, and $\left(\mathrm{f}_{\mathrm{u}}, \mathrm{f}_{\mathrm{v}}\right)=(1082.3,1073.7) \mathrm{px}$ (position of the optical center $\left(\mathrm{u}_{0}, \mathrm{v}_{0}\right)$ and the focal length in the $\mathrm{x}$ and $\mathrm{y}$ directions, respectively).

The system performance is evaluated in the next three subsections. The first one shows simulations experiments used in order to demonstrate the correct setup of the different parameters for the controller and the smoothing filter. The second experiment employs the real robot and camera in order to perform the tracking of a repetitive trajectory in the image space. This second subsection 
shows the correct tracking and the effect of solving the redundancy using the proposed cost function. The third subsection describes the behavior of the robotic system to detect abrupt changes during the tracking of faces in real experiments.

\subsection{Tracking of the desired trajectory without abrupt changes}

As previously indicated, the measurements obtained from the face detector are very noisy. The detector has considerable jitter in position that must be compensated using the proposed path tracker. This section describes simulation experiments in order to demonstrate the correct tracking of the image trajectory including the previous mentioned noise. To do this, the desired image trajectory represented in blue in Figure 3 is considered first. In this figure, the obtained measurements are indicated in green and with the symbol "*”. These simulation measurements have been manually introduced with considerable noise in order to observe the effect of the smoothing filter. Finally, the obtained image position estimations are indicated in red and with the symbol " $x$ ". For the image space task, the gain settings used are $\mathbf{K}_{\mathrm{P}}=\operatorname{diag}(0.1,0.1)$ and $\mathbf{K}_{\mathrm{D}}=\operatorname{diag}(0.5,0.5)$ (the proportional and derivative matrices). These values are considered in all the experiments presented in the result section. Additionally, the values of $\mathbf{Q}$ and $\mathbf{R}$ are experimentally determined and are considered to be $10^{-5} \cdot \mathbf{I}$ and $10^{-2} \cdot \mathbf{I}$, respectively.

Figure 3.a represents the trajectory obtained by using the proposed Kalman filter and Figure 3.b indicates the image trajectory obtained using the smoothing solution. As it can be seen in these figures, the correct tracking is achieved in both cases. However, using the smooth solution the estimated image positions are closer to the desired ones (see the marked zone in Figure 3). Thus, the estimations are less affected by the error introduced by the measurement algorithm. Additionally, to observe the improvements obtained by using the smoothing filter more clearly, the image error, $\mathbf{e}_{\mathrm{s}}=\mathbf{s}_{\mathrm{d}}-\mathbf{s}$, during the tracking is represented in Figure 4. Figure 4.a represents the image error obtained by using a Kalman filter and Figure 4.b the image error obtained by using the smoothing solution. In this last case, a lower error is obtained and the tracking behavior is less affected by the noise (an average image error of $1.97 \mathrm{px}$ is obtained by the Kalman filter and an average error of 1.14 is obtained by using the smoothing solution).

Once the effect of the proposed filter is observed in the image space, the correct behavior in the joint space is also demonstrated in the next section. 

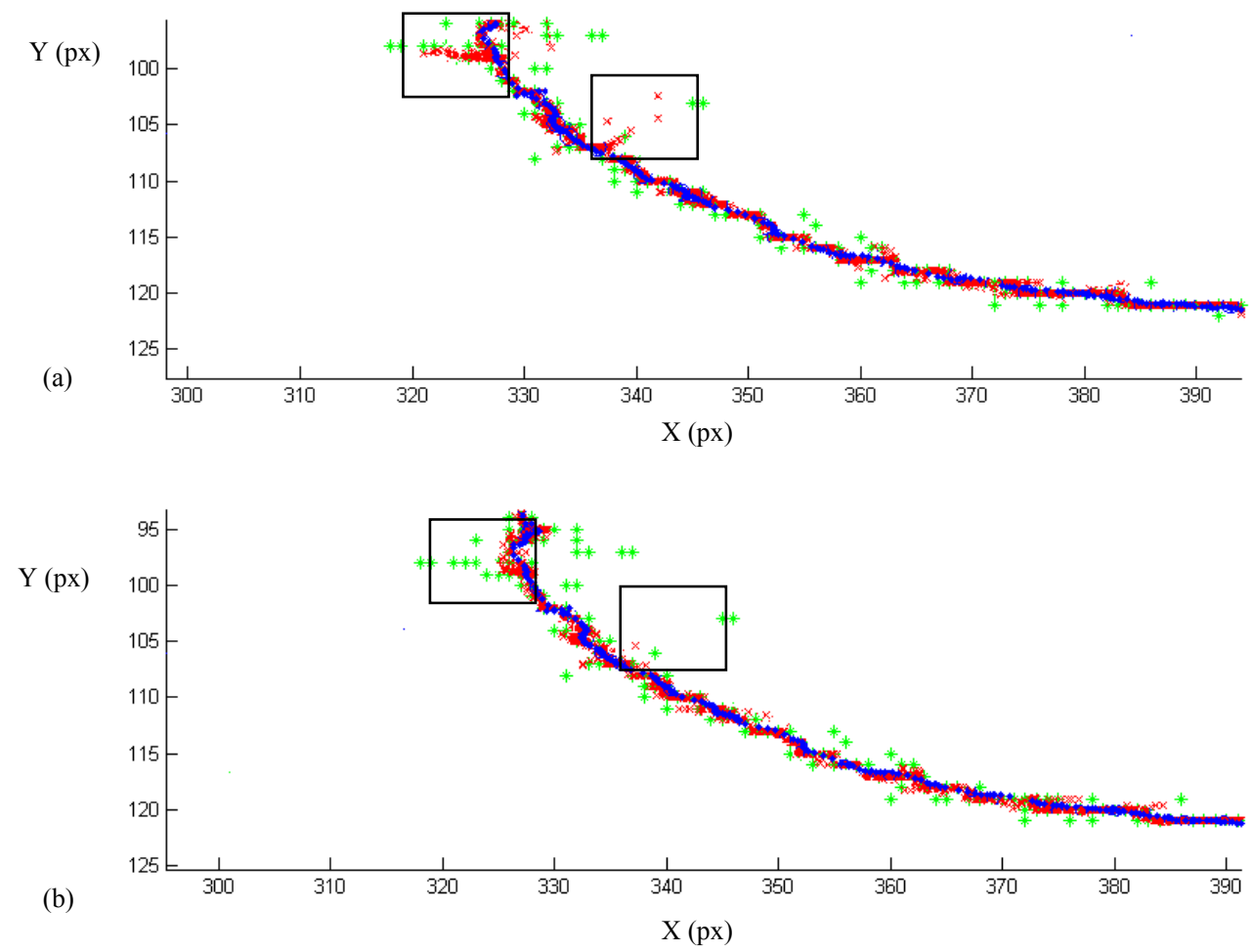

Figure 3. Image trajectory during the tracking. (a) Tracking obtained using the Kalman filter. (b) Tracking during the smoothing solution.

(a)

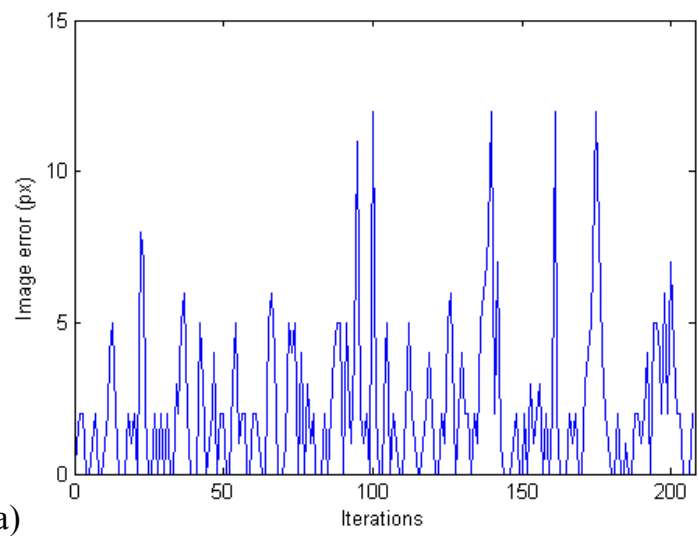

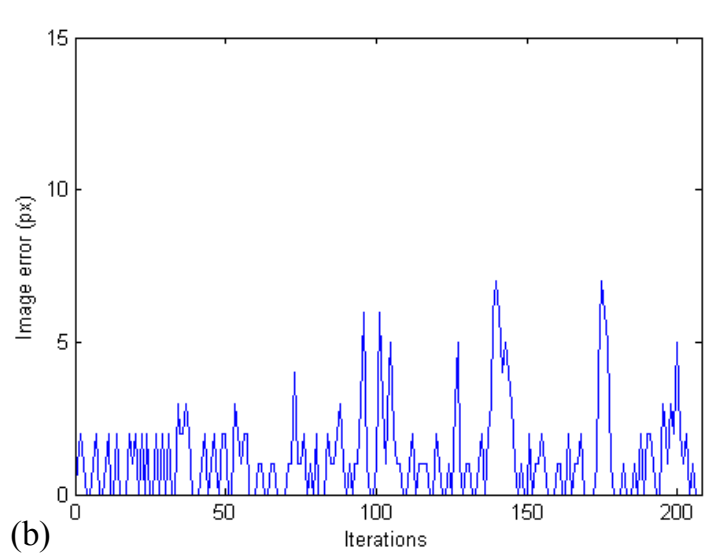

(b)

Figure 4. Evolution of the image error during the tracking. (a) Image error obtained using the Kalman filter. (b) Image error during the smoothing solution.

\subsection{Tracking of the desired trajectory without abrupt changes}

As previously described, the proposed visual servoing system is able to track time-dependent trajectories taking into account the robot dynamics. Additionally, the proposed visual control system can perform not only the tracking but also the robot postural control. To do this postural control, a secondary task is defined which is accomplished taking advantage of the robot redundancy. This 
section describes the results obtained during the tracking of a circular trajectory in order to show the improvement achieved by using this secondary task.

Let us consider the 7 dof robot carrying out the tracking of the desired image trajectory represented in Figure 5. To perform the experiment, the eye in hand camera is observing a visual feature $(\mathrm{k}=1)$ performing a planar movement with respect the robot at a distance of $50 \mathrm{~cm}$.

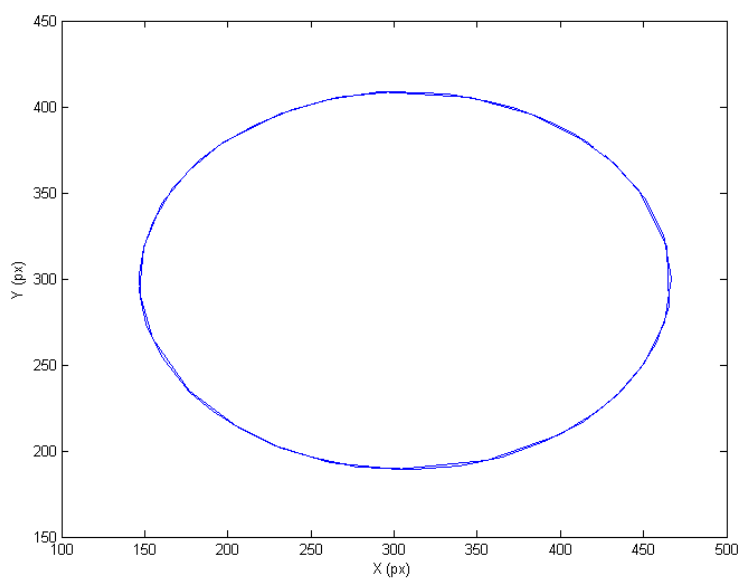

Figure 5. Desired image trajectory considered in the experiments.

Firstly, the system behavior without using the redundancy cost function is presented in Figure 6.a (only the primary task is considered). This figure represents the trajectory of the extracted image feature and the tracking error module in the image space $\left(\mathbf{e}_{\mathrm{s}}\right)$ when the desired image trajectory represented in Figure 5 is considered. Using the previous indicated parameters, the image error remains low and the repetitive trajectory is correctly tracked without using redundancy resolution. In Figure 6, there are no relevant differences between the use or not of the proposed redundancy resolution (Figure 6.b and Figure 6.a, respectively). In order to observe the difference, the robot trajectory in the joint space is represented in Figure 7 and Figure 8. Figure 7 represents the robot evolution in the joint space obtained using only the primary task and Figure 8 represents the joint trajectory employing both the primary and secondary tasks. Comparing both joint trajectories, it can be seen that a more oscillating behavior is obtained in Figure 7. Therefore, the use of the secondary task allows the robot to resolve the redundancy and a smoother behavior is obtained in the joint space by using the proposed cost function. 

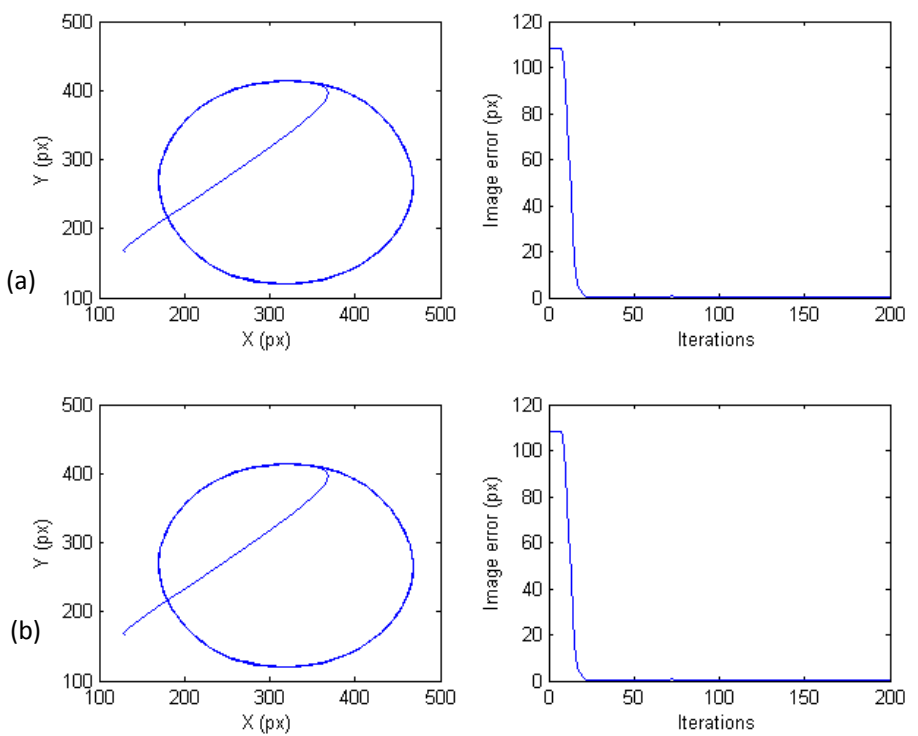

Figure 6. Tracking in the image space of the desired trajectory and error in the image: (a) Using the primary task. (b) Using the primary and secondary tasks.

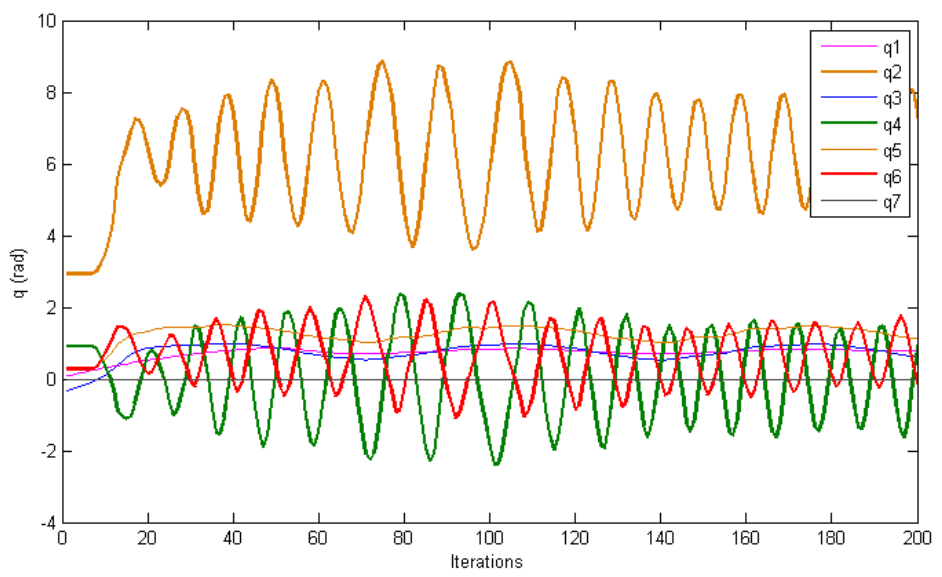

Figure 7. Robot joint coordinates during the tracking using the primary task.

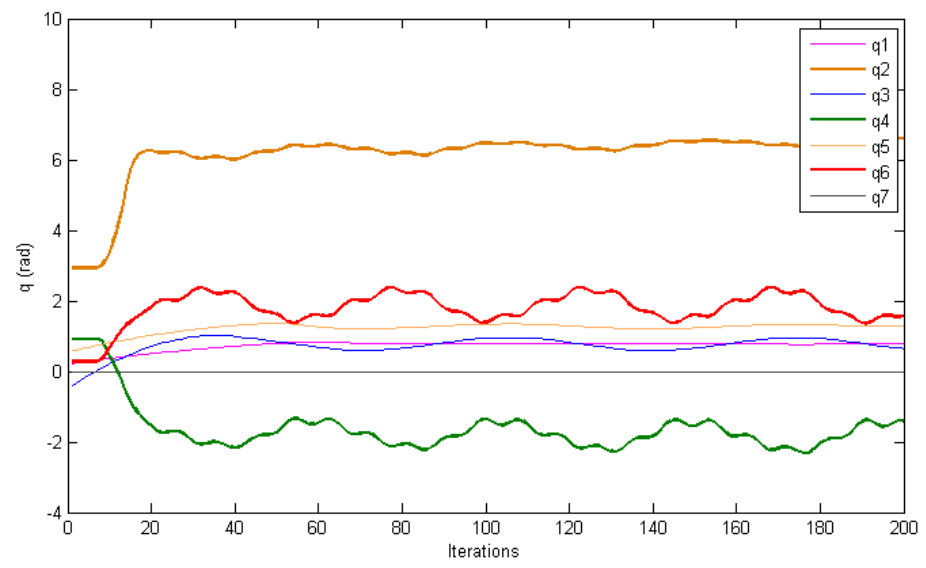

Figure 8. Robot joint coordinates during the tracking using the primary and secondary tasks. 


\subsection{Tracking the desired trajectory and detecting abrupt changes}

This section shows the tracking behavior of the proposed controller in real experiments. As it has been previously indicated, the direct controller must maintain the child's face within the camera field of view. Furthermore, due to the application requirements, the tracker must determine when abrupt changes occur during the tracking of the trajectory. These abrupt changes can indicate abnormal Asperger student's behavior. To do this, the ratio defined in Section 3.2 is employed.

Figure 9 represents the image measurements obtained during the first experiment in blue (only the $\mathrm{x}$ coordinate is represented). As it can be seen in this figure, noisy measurements are obtained. This last figure represents the trajectory obtained using the smoothing solution in red. The filtered image coordinates are less affected by the noise (the values of $\mathbf{Q}$ and $\mathbf{R}$ are $5 \cdot 10^{-6} \cdot \mathbf{I}$ and $2 \cdot 10^{-3} \cdot \mathbf{I}$, respectively). This trajectory presents an important abrupt change during iterations 115-130. This abrupt change is clearly detected by the proposed ratio in Figure 10. Figure 11 and Figure 12 represent another experiment. In this case, the student presents two abnormal behaviors which must be detected (iterations 60-90 and 125). As it can be seen in Figure 12, the ratio clearly detects the abrupt changes independently of the noise in the obtained measurements. This last experiment also presents (iteration 150) a normal change in the face trajectory. This is not an abrupt change and it is a normal behavior during the experiment. As expected, the ratio in Figure 12 has a low value during this change.

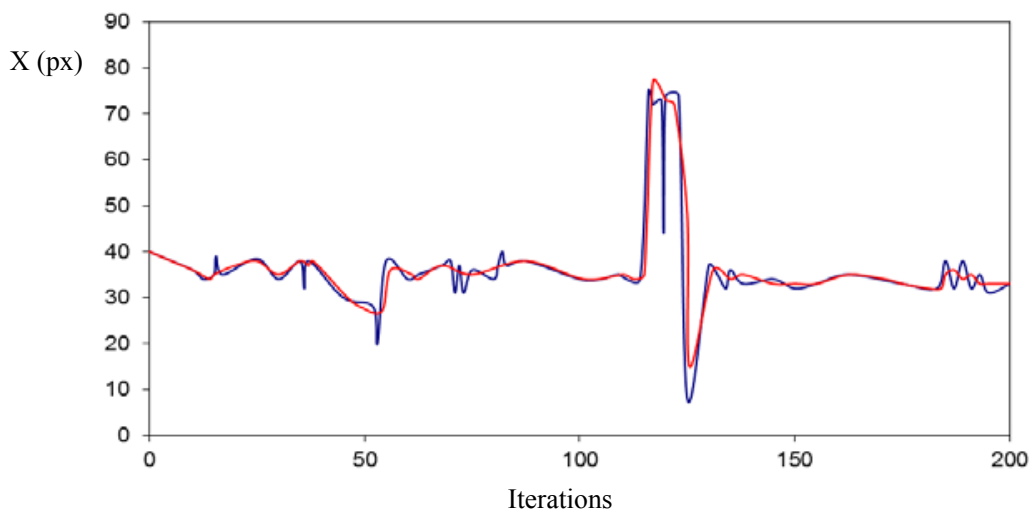

Figure 9. X coordinate in the image of the tracked face during the first experiment. The measurements obtained are represented in blue. The image $\mathrm{X}$ coordinates obtained from the smoothing solution are represented in red. 


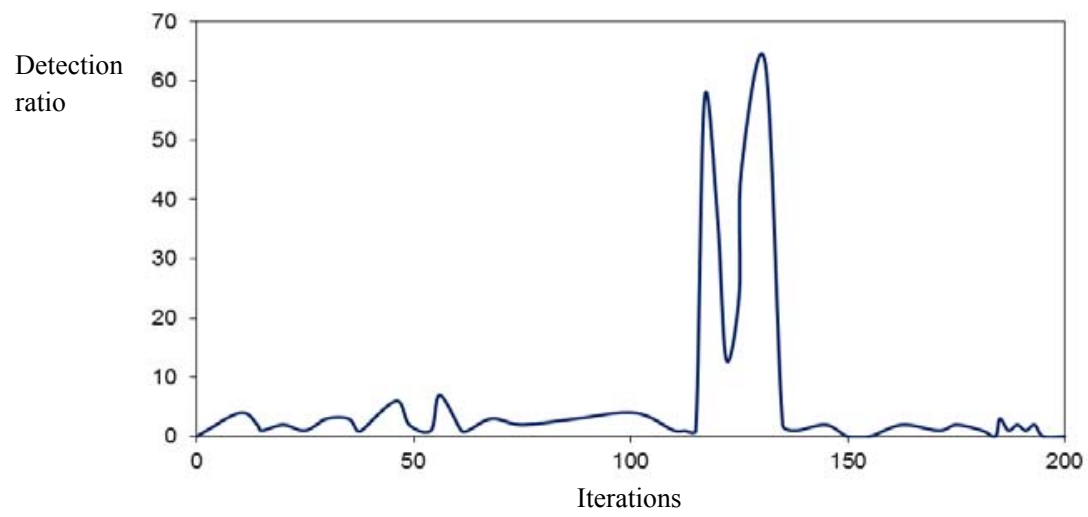

Figure 10. Ratio obtained from the smoothing solution represented in Figure 9.

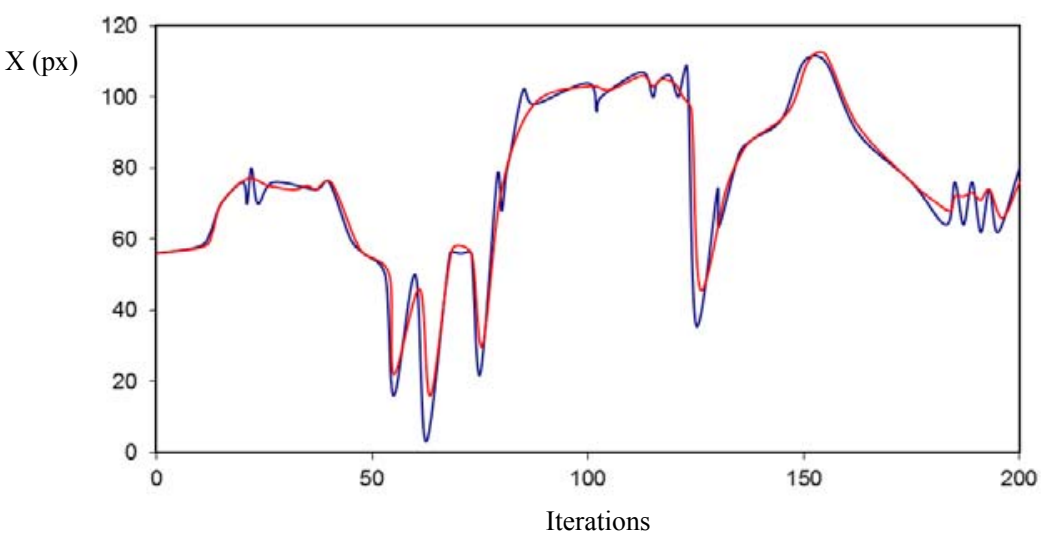

Figure 11. X coordinate in the image of the tracked face during the second experiment. The measurements obtained are represented in blue. The image X coordinates obtained from the smoothing solution are represented in red.

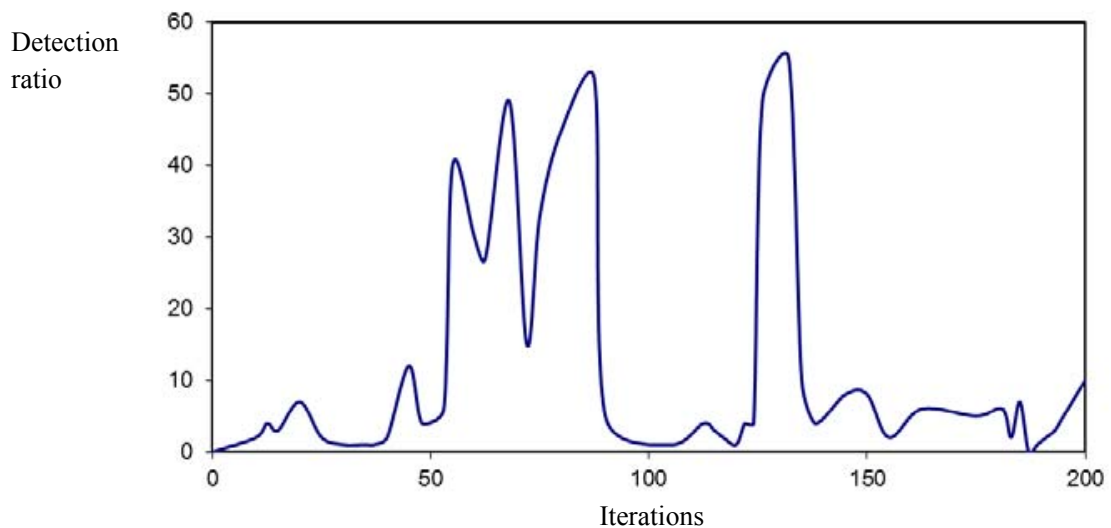

Figure 12. Ratio obtained from the smoothing solution represented in Figure 11. 


\section{Conclusions}

The use of visual servoing systems permits the robot guidance with respect to an observed object using image information. To do this, the classical image-based controllers determine the required end-effector robot velocities in order to position the robot with respect to a reference object. In our approach, a direct visual controller which generates the required joint torques is proposed in order to perform the tracking in the image space. The proposed controller presents several contributions which are illustrated in the result section. Firstly, the visual servo controller generates directly the required torques, in order to accomplish prioritized tasks. This last aspect guarantees that the system reacts more quickly to abrupt changes in the trajectory in comparison to classical visual servoing systems. Furthermore, this controller takes advantage of the redundancy in order to guarantee not only the correct tracking of the image trajectory but also a secondary task. As it has been illustrated in the result section, a smoother behavior is obtained using the proposed redundancy resolution. This last aspect guarantees less stress in the joints.

Another important aspect of the proposed controller is the possibility to continuously filter the measurements and to determine the moment when these measurements do not fulfill the motion model employed by the filter. This last aspect is used not only to detect abrupt changes in the trajectory performed by the child's face but also to estimate and compensate these changes using the proposed filter. All the contributions have been integrated in an immersive virtual reality system in order to improve the social skills of Asperger students. Although, the pedagogical aspects are not included in this paper, the different controllers proposed in the paper have successfully been applied in order to improve the social skills of these children.

Currently we are working with the use of cooperative robots in order to better track the child's face from different points of view. This last aspect will increase the tracking robustness with respect to occlusions.

\section{Acknowledgements}

We would like to thank the Intelligent Systems Group, Electronic Imaging Department of the Fraunhofer Institute for Integrated Circuits IIS for their cooperation.

\section{References}

[1] Schiffer, S., Ferrein, A., Lakemeyer, G.: Caesar: an intelligent domestic service robot, Journal of Intelligent Service Robotics, 5(4), 259-273, (2012). 
[2] Chaumette, F., Hutchinson, S.: Visual Servo Control, Part I: Basic Approaches, IEEE Robotics and Automation Magazine, 13(4), 82-90, (2006).

[3] Pomares, J., Corrales, J. A., Garcia, G. J., Torres, F.: Direct Visual Servoing to Track Trajectories in Human-Robot Cooperation, International Journal of Advanced Robotic Systems, 8(4), 129-138, (2010).

[4] Garcia, G. J., Corrales, J. A., Pomares, J., Torres, F.: Survey of Visual and Force/Tactile Control of Robots for Physical Interaction in Spain, Sensors, 9(12), 9689-9733, (2009).

[5] Farooq, M., Wang, D. B.: Hybrid force/position control scheme for flexible joint robot with friction between and the end-effector and the environment, International Journal of Engineering Science, 46(12), 1266-1278, (2008).

[6] Kalman, R. E.: A New Approach to Linear Filtering and Prediction Problems, Transactions of the ASME-Journal of Basic Engineering, 82(D), 35-45, (1960).

[7] Simon, D.: Kalman Filtering. Embedded Systems Programming, 14(6), 72-79, (2001).

[8] Foxlin, E.: Pedestrian Tracking with Shoe-Mounted Inertial Sensors, IEEE Computer Graphics and Applications, 25(6), 38-46, (2005).

[9] Roetenberg, D., Slycke, P. J., Veltink, P. H.: Ambulatory Position and Orientation Tracking Fusing Magnetic and Inertial Sensing, IEEE Transactions on Biomedical Engineering, 54(5), 883890, (2007).

[10] Caron, F., Duflos, E., Pomorski, D., Vanheeghe, P.: GPS/IMU Data Fusion Using Multisensor Kalman Filtering: Introduction of Contextual Aspects, Information Fusion, 7(2), 221-230, (2006).

[11] Ribo, M., Brandner, M., Pinz, A.: A Flexible Software Architecture for Hybrid Tracking, Journal of Robotic Systems, 21(2), 53-62, (2004).

[12] Silveira, G., Archer, R.: Direct Visual Servoing: Vision-Based Estimation and Control Using Only Nonmetric Information, IEEE Transactions on Robotics, 28(4), 974-980, (2012).

[13] Bertozzi, M., Broggi, A., Fascioli, A.: Vision-based intelligent vehicles: State of the art and perspectives, Robotics and Autonomous Systems, 32(1), 1-16, (2000).

[14] Royer, E., Bom, J., Dhome, M., Thuilot, B., Lhuillier, M., Marmoiton, F.: Outdoor autonomous navigation using monocular vision, 2005 IEEE/RSJ International Conference on Intelligent Robots and Systems, Aug. 2005, pp.1253,1258.

[15] Pomares, J., Torres F.: Movement-flow-based visual servoing and force control fusion for Manipulation Tasks in unstructured environments. IEEE Transactions on Systems, Man, and Cybernetics, Part C 35(1): 4-15 (2005).

[16] Mezouar, Y., Chaumette, F.: Path planning for robust image-based control. IEEE Transactions on Robotics and Automation 18(4): 534-549 (2002).

[17] Garcia, G.J., Jara, C.A., Pomares, J., Torres, F.: Direct visual servo control of a robot to track trajectories in supervision tasks. 11th International Conference on Control Automation Robotics \& Vision (ICARCV), 2010, pp.1434 - 1439.

[18] Pomares, J., Candelas, F. A., Torres, F., Corrales, J. A., Garcia, G.J.: Safe human-robot cooperation based on an adaptive time-independent image path tracker. International Journal of Innovative Computing, Information and Control 6(9): 3819-3842 (2010).

[19] Cheah, C.C., Liu, C., Slotine, J.E.: Adaptive Vision based Tracking Control of Robots with Uncertainty in Depth Information. 2007 IEEE International Conference on Robotics and Automation, April 2007, pp.2817,2822. 
[20] De Luca, A., Ferri, M., Oriolo, G., Giordano, P. R.: "Visual Servoing with Exploitation of Redundancy: An Experimental Study" 2008 IEEE International Conference on Robotics and Automation. Pasadena, CA, USA, May, 2008, pp. 3231 - 3237.

[21] Kumar, S., Premkumar, P., Dutta, A., Behera, L.: Visual motor control of a 7DOF redundant manipulator using redundancy preserving learning network, Robotica, 28, 795-810, (2010).

[22] Mansard, N., Chaumette F.: "A new redundancy formalism for avoidance in visual servoing" IEEE/RSJ International Conference on Intelligent Robots and Systems, Edmonton, Canada August, 2005, pp. $1694-1700$.

[23] Sawo, F., Fujita, M., Sawodny, O.: Passivity-based dynamic visual feedback control of manipulators with kinematic redundancy. 2005 IEEE Conference on Control Applications, August, 2005, pp.1200-1205.

[24] Trujano, M. A., Garrido, R., Soria, A.: Stable Visual PID Control of Redundant Planar Parallel Robots, in PID Control, Implementation and Tuning, T. Mansour, Ed. InTech, 2011, pp. 27 - 50.

[25] Pomares, J., Perea, I., García, G.J., Jara, C.A., Corrales, J.A., Torres, F.: A multi-sensorial hybrid control for robotic manipulation in human-robot workspaces. Sensors (Basel), 11(10), 9839-9862, (2011).

[26] Correa, M., Hermosilla, G., Verschae, R., Ruiz-del-Solar, J.: Human Detection and Identification by Robots using Thermal and Visual Information in Domestic Environments. Journal of Intelligent and Robot Systems 66, 223-243, (2012).

[27] Lorenzo, G., Pomares, J., Lledó, A.: Inclusion of immersive virtual learning environments and visual control systems to support the learning of students with Asperger syndrome. Computers \& Education 62, 88-101, (2013).

[28] Ernst, A., Ruf, T., Küblbeck, C.: A modular framework to detect and analyze faces for audience measurement systems. 2nd Workshop on Pervasive Advertising 2009, Lübeck, Germany: 75-87, 2009.

[29] Ertürk, S.: Real-Time Digital Image StabilizationUsing Kalman Filters. Real-Time Imaging 8, 317-328, (2002).

[30] Lippiello, V., Siciliano, B., Villani, L.: Visual motion tracking with full adaptive extended Kalman filter: an experimental study, $16^{\text {th }}$ IFAC World Congress, Prague Czech Republic, July, 2005.

[31] Gelb, A.: Applied Optimal Estimation, MIT Press, Cambridge, MA, 1974. 